OPEN ACCESS

Edited by:

Changbin Chen,

University of Minnesota, USA

Reviewed by:

Penny M. A. Kianian, University of Minnesota, USA

Chengdao Li,

Murdoch University, Australia

*Correspondence:

Manuela Nagel

Nagel@ipk-gatersleben.de

Specialty section:

This article was submitted to

Plant Genetics and Genomics,

a section of the journal

Frontiers in Plant Science

Received: 13 December 2015 Accepted: 14 March 2016

Published: 31 March 2016

Citation:

Nagel M, Kodde J, Pistrick S, Mascher M, Börner A and Groot SPC (2016) Barley Seed Aging: Genetics behind the Dry Elevated Pressure of Oxygen Aging and Moist Controlled Deterioration. Front. Plant Sci. 7:388. doi: $10.3389 / f p / s .2016 .00388$

\section{Barley Seed Aging: Genetics behind the Dry Elevated Pressure of Oxygen Aging and Moist Controlled Deterioration}

\author{
Manuela Nagel ${ }^{1 *}$, Jan Kodde ${ }^{2}$, Sibylle Pistrick ${ }^{1}$, Martin Mascher ${ }^{1}$, Andreas Börner ${ }^{1}$ and \\ Steven P. C. Groot ${ }^{2}$ \\ 1 Genebank Department, Leibniz Institute of Plant Genetics and Crop Plant Research (IPK Gatersleben), Stadt Seeland, \\ Germany, ${ }^{2}$ Wageningen UR, Plant Research International B.V., Wageningen, Netherlands
}

Experimental seed aging approaches intend to mimic seed deterioration processes to achieve a storage interval reduction. Common methods apply higher seed moisture levels and temperatures. In contrast, the "elevated partial pressure of oxygen" (EPPO) approach treats dry seed stored at ambient temperatures with high oxygen pressure. To analyse the genetic background of seed longevity and the effects of seed aging under dry conditions, the EPPO approach was applied to the progeny of the Oregon Wolfe Barley (OWB) mapping population. In comparison to a non-treated control and a control high-pressure nitrogen treatment, EPPO stored seeds showed typical symptoms of aging with a significant reduction of normal seedlings, slower germination, and less total germination. Thereby, the parent Dom (“OWB-D"), carrying dominant alleles, is more sensitive to aging in comparison to the population mean and in most cases to the parent Rec (“OWB-R”), carrying recessive alleles. Quantitative trait locus (QTL) analyses using 2832 markers revealed 65 QTLs, including two major loci for seed vigor on $2 \mathrm{H}$ and $7 \mathrm{H}$. QTLs for EPPO tolerance were detected on $3 \mathrm{H}, 4 \mathrm{H}$, and $5 \mathrm{H}$. An applied controlled deterioration (CD) treatment (aged at higher moisture level and temperature) revealed a tolerance QTL on $5 \mathrm{H}$, indicating that the mechanism of seed deterioration differs in part between EPPO or CD conditions.

Keywords: caryopsis, seed conservation, seed storage, genotype, germination, linkage mapping

\section{INTRODUCTION}

Knowledge of the mechanism of seed longevity has become important since international companies have started to ship seeds around the world and gene banks initiated the first seed collections at the beginning of the twentieth century. Thereby, long-term survival is greatly influenced by plant breeders who continuously work on seed quality performance.

Desiccation-tolerant (orthodox) seeds extend their life span when they reach maturity, dry, and enter the glassy state (Walters et al., 2005a; Buitink and Leprince, 2008). A glass is an amorphous, solid state with high viscosity (Buitink and Leprince, 2004) where metabolic processes are reduced to a minimum or can be even excluded (Kranner et al., 2010; Fernández-Marín et al., 2013). In this state, reactive oxygen species (ROS; Bailly, 2004; El-Maarouf-Bouteau et al., 2011), non-enzymatic Amadori and Maillard reactions (Sun and Leopold, 1995), and lipid peroxidations 
can damage macromolecules such as DNA, proteins, and lipids, and lead to seed deterioration. Further extrinsic factors can be multifaceted and include abiotic and biotic stress during seed development, seed maturity, harvest, drying, and cleaning. The rate of decay depends largely on the storage environment, as temperatures (Roberts, 1973), atmosphere (Groot et al., 2014) and relative humidity (RH) interact with the genotype (Walters et al., 2005b; Nagel and Börner, 2010). Consequently, a temperature or $\mathrm{RH}$ increase cause a viscosity decrease and thus a glass to rubber state change with higher molecular mobility and reaction kinetics (Walters, 1998).

The investigation of seed deterioration under natural conditions is protracted, as optimally stored orthodox seeds can survive for decades without any effect on the germination. To study seed deterioration more quickly, experimental approaches were invented by Roberts (1960) and refined by Hampton and Tekrony (1995) and Hay et al. (2008). Basically, temperatures are adjusted to above $30^{\circ} \mathrm{C}$ and seed moisture levels are increased, which converts the glassy state into a rubber state, with dramatic effects on chemical reactions (Walters, 1998). These methods have been widely applied for investigations on ecological (Probert et al., 2009), molecular (Waterworth et al., 2010), biochemical (Lehner et al., 2008), and genetic (Sasaki et al., 2015) levels. However, biochemical processes of artificially aged seeds in the rubber state can deviate from those in industrial dry storage at ambient temperatures or in cold, dry storage in gene banks, such as the half-reduction potential of the antioxidant glutathione and an assumed shift in $\mathrm{pH}$ (Nagel et al., 2015). Therefore, Groot et al. (2012) developed the "elevated partial pressure of oxygen" (EPPO) approach, which avoids a glass-rubber transition while increasing oxidation by exposing the dry seeds to high oxygen pressure (up to $18 \mathrm{MPa}$ ). This treatment affects germination in such a way that the visual appearance of seed deterioration is consistent with that observed after long term dry storage.

So far, the genetic backgrounds of seed longevity have been investigated by exposing seeds to relatively moist experimental aging conditions. Quantitative trait locus (QTL) analysis and association mapping revealed that longevity QTLs often colocated with QTLs for other traits, including morphological traits such as spike compactness (Rehman Arif et al., 2012). In barley, whose seeds are categorized as having medium-long storage potential (Walters et al., 2005b), QTLs for longevity colocated with QTLs for plant height and naked caryopsis on two chromosomes (Nagel et al., 2009).

The aim of this study was to investigate the genetic background of barley seed longevity by applying the EPPO treatment to dry-stored seeds. Double haploid $(\mathrm{DH})$ lines

\footnotetext{
Abbreviations: AUC, Area under the curve; BLASTX, Basic Local Alignment Tool; C08, Control of CD experiment in 2008; CD, Controlled deterioration; CD08, Controlled deterioration experiment in 2008; DArT, Diversity Array Technology; DH, Double haploid; E, Expect Value; EPPO, Elevated partial pressure of oxygen; EST, Expressed sequence tag; LOD, Logarithm of odds; OWB, Oregon Wolfe Barley; “OWB-D," Dominant parent Dom; “OWB-R,” Recessive parent Rec; P50, Half-viability period; QTL, Quantitative trait locus; $\mathrm{R}^{2}$, Explained phenotypic variation; RH, Relative humidity; ROS, Reactive oxygen species; TC, Treatment Control of EPPO experiment in 2011; T50, Time to 50\% germination; \%NS, Percentage of normal seedlings; \%TG, Percentage of total germination.
}

of the Oregon Wolfe Barley (OWB) mapping population, genotyped with 2383 markers (Chutimanitsakun et al., 2011), were equilibrated to $40 \% \mathrm{RH}$ at $20^{\circ} \mathrm{C}$ and exposed to high oxygen pressure at $18 \mathrm{MPa}$. Control and deterioration effects after treatment were assessed by germination speed and compared with previous experiments using CD (Nagel et al., 2009). Both data sets were analyzed and reanalyzed with the currently available marker sets and differences after QTL mapping are discussed.

\section{MATERIAL AND METHODS}

\section{Experimental Material}

Caryopses, henceforth termed seeds, of the DH OWB mapping population were studied on their ability to cope with stress during storage. The set of 94 spring barley lines was developed by pollinating the Hordeum vulgare $\mathrm{F} 1$ hybrid produced by the dominant parent Dom ("OWB-D") $\times$ recessive parent Rec (“OWB-R") with $H$. bulbosum (Costa et al., 2001). Characteristics of "OWB-D" and "OWB-R" were selected as dominant and recessive morphological marker stocks (Wolfe and Franckowiak, 1991), whereby more information can be found at http://barleyworld.org/oregonwolfe.php. A marker set of 2832 markers including 463 Restriction site Associated DNA (RAD) markers was developed by Chutimanitsakun et al. (2011) and is available at http://wheat.pw.usda.gov/ggpages/maps/OWB/. In 2005, a large number of seeds were produced under optimum conditions to maximize seed yield and quality in Gatersleben, Germany, and stored in conditions of $10 \%$ seed moisture content and $-18^{\circ} \mathrm{C}$.

\section{Storage Treatment}

The EPPO treatment was applied at Wageningen UR in 2011 according to Groot et al. (2012). Seeds of the OWB population were equilibrated to $40 \% \mathrm{RH}$ at $20^{\circ} \mathrm{C}$ for 2 weeks. Thereafter, the seeds were stored under ambient atmospheric pressure in 11 rubber-sealed glass jars (TC, treatment control) or in 121 steel tanks under an elevated partial pressure of oxygen (EPPO) or nitrogen (EPPN) gas. The latter were used to control for potential damage induced by the high pressure and/or pressure release from the high pressure storage. The seed samples were placed within the tanks in $13 \mathrm{ml}$ polystyrene tubes (Sarstedt, Germany) perforated with holes of $\sim 1 \mathrm{~mm}$ diameter and closed with low-density polyethylene push caps. The tanks were filled slowly ( $\sim 6 \mathrm{bar} / \mathrm{min}$ ) with oxygen or nitrogen from large buffer tanks, until the tank pressure reached $\sim 18 \mathrm{MPa}$. To minimize temperature changes during filling, the tanks were placed in water at ambient temperature. Filling of the tanks with oxygen was performed by skilled staff at a local scuba diving shop (4Divers, Veenendaal, The Netherlands). As tanks were not flushed before filling, they retained the initial atmospheric amounts of oxygen (0.021 MPa partial pressure), nitrogen and other minor gases present in air. Silica gel, equilibrated to $40 \% \mathrm{RH}$, was also added to the tanks and glass jars to buffer RH in the containers. The tank pressure was released with an average relative pressure decline of $0.5 \%$ per min (maximum $3.0 \%$ ) using a computer-controlled relative flow rate. Between experiments and prior to germination 
testing, seeds were stored in a cabinet with air circulating above a saturated $\mathrm{CaCl}_{2}$ salt solution ( $\mathrm{RH} 35 \%$ ) at a temperature of $20^{\circ} \mathrm{C}$. Seeds were packaged in sealed laminated foil bags and sent to IPK Gatersleben. As an initial test, 10 lines of the "W766" barley population, multiplied and stored together with seeds of the OWB population, were treated for 4, 6, 8, and 12 weeks in oxygen at $18 \mathrm{MPa}$ (atmospheric pressure is $\sim 0.1 \mathrm{MPa}$ ) and showed a significant reduction in germination and germination speed between 8 and 12 weeks (Supplementary Figure 1). For the main experiment, the seeds were stored for 9 weeks under EPPO, EPPN, or ambient pressure conditions (TC).

\section{Germination Test}

At IPK Gatersleben, seeds were subjected to germination tests. Four replicates of 50 seeds (for a few lines, only 10-40 seeds per replicate were available) were placed on moistened filter paper and kept in a germination chamber at $20^{\circ} \mathrm{C}, 60 \% \mathrm{RH}$ for $8 \mathrm{~h}$ of light. Germinating seeds were determined daily by examining radicle protrusion. After a period of 10 days, seeds were classified into normal seedlings, which show the potential to develop into satisfactory plants; abnormal seedlings, which are damaged, deformed, or decayed, and do not show potential to develop into a normal plant; and non-germinating seeds according to (ISTA, 2012). The percentage of total germinated seeds (\%TG) and normal seedlings (\%NS), the time to reach $50 \%$ of the maximum germination (T50), and the area under the curve (AUC) after $100 \mathrm{~h}$ were calculated using the curve-fitting module of the Germinator (Joosen et al., 2010).

\section{Controlled Deterioration}

In 2008, DH lines of the OWB population were experimentally aged at $44^{\circ} \mathrm{C}$ and $18 \%$ seed moisture content, following the procedure of CD by Hampton and Tekrony (1995). Four replicates of 50 seeds [control of 2008 tests (C08) and controlled deteriorated seeds of 2008 tests (CD08)] were germinated according to ISTA (2008) and published in Nagel et al. (2009). Here, \%NS and \%TG were recalculated and, on the basis of viability equation $v=K_{i}-\sigma^{-1} \mathrm{p}$ of Roberts (1973), the halfviability period (P50) for CD and EPPO aging was analyzed using $\%$ NS for initial seed germination $\left(K_{i}\right)$ and 63 days and 3 days to calculate sigma $(\sigma)$ for EPPO and CD, respectively. Further, \%NS were transformed to probit units and used to subtract control and EPPN from the EPPO and CD treatments and to overcome the effect of the different initial germinations of the genotypes. This resulted in additional traits for QTL mapping: 3-year storage (C08-TC), TC-EPPN, EPPO-TC, EPPO-EPPN, CD08-TC08.

The Shapiro-Wilk test indicated that, except for T50, all data did not match the pattern produced by a population with normal distribution. Therefore, Friedman Repeated Measures Analysis of Variance on Ranks, followed by Tukey's and Dunn's tests, was used to analyse differences $(P<0.05)$ between treatments.

\section{QTL Mapping}

QTL analyses for each of the phenotypic characters were conducted for the 93 OWB lines using the composite interval mapping procedure Zeng (1994) implemented in Windows QTL Cartographer 2.5 (Wang et al., 2011). Experiment-wise significance likelihood ratio test (LR) statistic thresholds ( $P$ $\leq 0.05)$ for QTL identification were determined with 1000 permutations, expressed as logarithm of odds (LOD $=0.217 \mathrm{LR})$, and reinforced the set LOD threshold of 3. Significant QTL were characterized by the position of significant flanking markers, the marker with highest LOD, the proportion of phenotypic variance explained by the individual QTL $\left(R^{2}\right)$, the additive effect (expressed as one-half of the difference between the two allelic classes), and the LOD threshold. Negative values indicate that alleles were contributed by the parent "OWB-R" and positive values by the parent "OWB-D."

\section{Gene Content in QTL Regions}

Sequences of flanking markers of QTL regions were mapped to the whole-genome shotgun assembly of barley cv. "Morex" (The International Barley Genome Sequencing Consortium, 2012) with BWA mem version 0.7.12 (Li, 2013). Primary alignments with mapping quality $\geq 30$ were extracted with SAMtools (Li et al., 2009), converted to BED format with BEDTools (Quinlan and Hall, 2010) and imported into R (R Core Team, 2015). Genetic positions of QTL regions in the POPSEQ genetic map (Mascher et al., 2013) were determined from the alignments of marker sequences to the Morex WGS contigs anchored by POPSEQ. If both flanking markers of a QTL regions were positioned in the POPSEQ map, the identifiers and functional annotation of all genes (The International Barley Genome Sequencing Consortium, 2012) between flanking markers were exported into a spreadsheet using functionalities of the R packages "openxlsx" (https://cran.r-project.org/web/packages/openxlsx/) and "data.table" (https://cran.r-project.org/web/packages/data.table/).

\section{RESULTS}

\section{Phenotypic Data}

Population lines were selected as dominant and recessive morphological marker stocks and show high phenotypic diversity. After 6 years of storage at $10 \%$ seed moisture content and $-18^{\circ} \mathrm{C}$, the seed material had on average $86.2 \pm 16.3 \% \mathrm{NS}$ and differed significantly between "OWB-D" (54.0\%) and "OWBR” (92.5\%; Supplementary Figure 2). These differences were also reflected in the AUC and T50, which showed lower area and longer time to reach $50 \%$ germination for "OWB-D" (AUC = 35.8; $\mathrm{T} 50=49.2 \mathrm{~h})$ in comparison to "OWB-R" (AUC = 72.3; T50 = 27.6 h; Figure 1 and Supplementary Table 1). Except for T50 after EPPO and for \%TG and P50 after CD08, the parent "OWB-D" showed worse performance than the mean of the population. "OWB-R" was only inferior to the mean of population after EPPO treatment.

To compensate for potential damage induced by high pressure and pressure release, high nitrogen pressure (EPPN) was applied to seeds of the OWB population. The \%NS was the only trait that showed a significant $(P<0.05)$ effect between both controls, storage at ambient pressure and EPPN treatment (Supplementary Table 1). In contrast, a high oxygen concentration, as applied in the EPPO treatment, affected germination significantly $(P<$ $0.05)$, resulting in a reduced $\% \mathrm{NS}, \% \mathrm{TG}$, and AUC and extended 


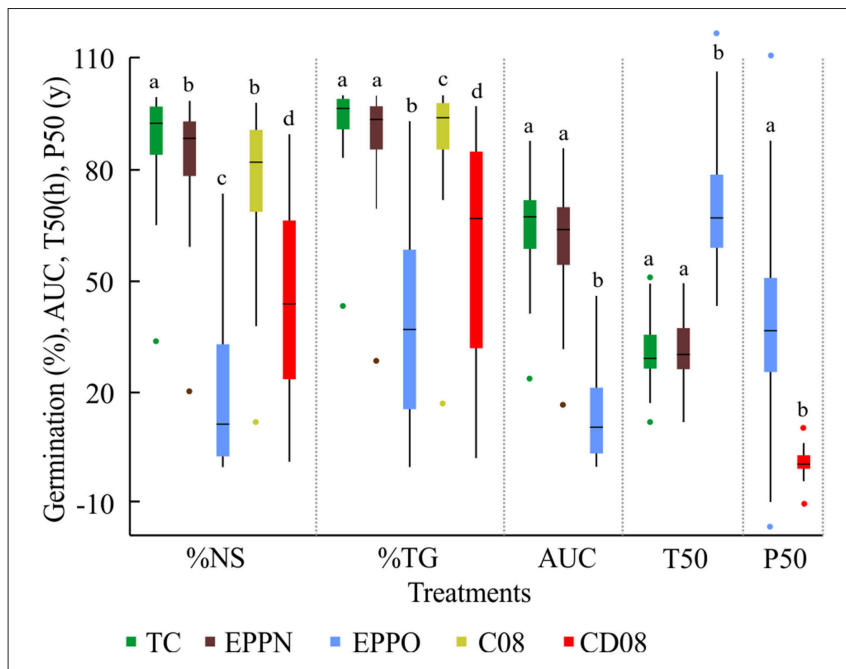

FIGURE 1 | Germination performance of 94 Oregon Wolfe Barley (OWB) lines after different storage treatments tested in 2008 and 2011.

Germination performance is expressed as the percentage of normal seedlings (NS\%), total germination (TG\%), area under the curve (AUC), time to 50\% germination (T50 in hours), and half-viability period (P50 in days). Boxplots show minimum and maximum values (spots), 25\% and $75 \%$ quartiles, and whiskers of the 1.5 interquartile range. Color code is given in the figure. C08, control performed in 2008; CD08, controlled deterioration performed in 2008 EPPN, elevated partial pressure of nitrogen storage; EPPO, elevated partial pressure of oxygen storage; TC, treatment control at ambient air pressure. a, b, c, and d symbolize significant differences between treatments at $P<0.05$.

T50 (Figure 1). Seedling quality differed between the treatments and was best in the control. After the high-pressure nitrogen treatment, there was a minor relative increase in abnormal seedlings with no shoot, white shoots, or deformations, and of non-germinated seeds, resulting in an $\sim 5 \%$ decline in normal seedlings in comparison to the control seeds stored under ambient pressure conditions (Figure 2). The EPPO treatment caused extensive damage which resulted in a relative increase in seedlings with no shoot, no root, white shoots, deformation, and no germination of $190,1026,1600,235$, and $587 \%$, respectively.

Comparing the 9 weeks EPPO treatment with the CD08 treatment, less damage was observed after the CD08 treatment. Only 38\% more abnormal seedlings (not differentiated between white, deformed, no root and no shoot seedlings) were Counted (Figure 2). After 3 days of CD, CD08 was significantly different $(P<0.05)$ to control in 2008 and to EPPO treatment in 2011, and revealed an average decline in \%NS and \% TG of OWB population to 44.3 and $60.3 \%$, respectively. Again, with some exceptions, the parent "OWB-D" performs less well than the population mean in all traits whereas "OWB-R" revealed higher initial germination but comparable germinations after CD.

On the basis of \%NS in the ambient control, EPPN, and after EPPO, the P50 was calculated and resulted in a mean for the whole population of $38.5 \pm 21.8$ days after the EPPO treatment and of $2.3 \pm 3.5$ days after CD08 treatment. As demonstrated by the previous data, the parent "OWB-D" has a shorter half-viability period in comparison with "OWB-R" and

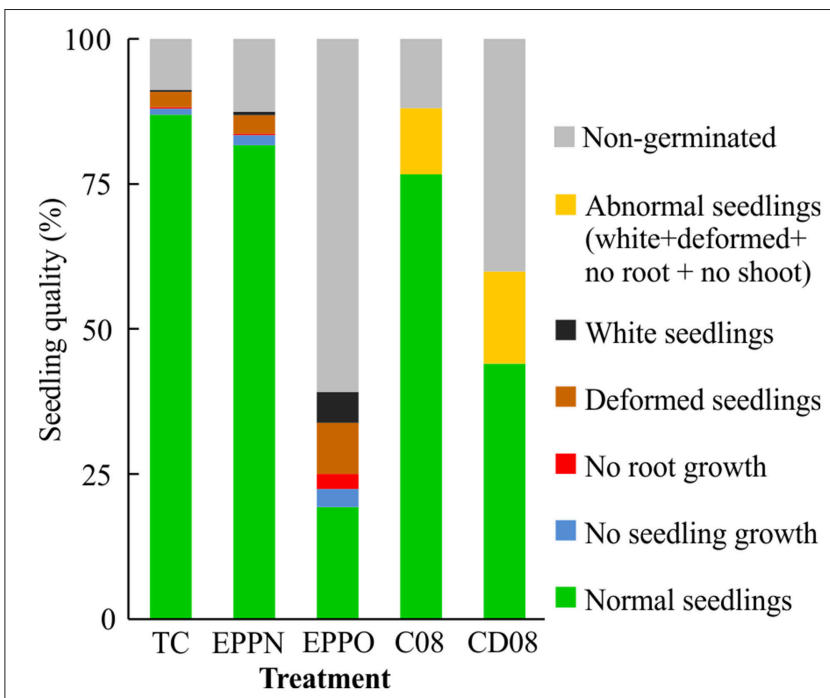

FIGURE 2 | Seedling quality of Oregon Wolfe Barley (OWB) lines after storage under atmospheric pressure (TC, treatment control), high-pressure nitrogen (EPPN), and high-pressure oxygen (EPPO). For comparisons, normal, abnormal (not discriminated into white, deformed, no root, no seedling growth), and non-germinated seedlings of C08 (control performed in 2008) and CD08 (controlled deterioration performed in 2008) are shown.

the mean half-viability period of the $\mathrm{DH}$ population was higher than that of either parent.

Correlation analysis revealed that EPPN treatment showed a higher correlation with control treatments than did EPPO treatment. Here, the highest coefficients $(r=-0.62 ; P<0.001)$ were found between \%TG of EPPO and T50 of controls, and indicate a weak relationship between both. All the germination parameters measured showed highly significant correlations $(P<$ 0.001 ) with controls following EPPN treatment but this was not so following EPPO treatment, when not all parameters correlated significantly and those that did had lower r values (Table 1).

\section{QTL Mapping}

In total, 2832 markers were used to analyse the EPPO experiments and to reanalyse the results of the 2008 experiments (Figure 3; Table 2). In total, 65 significant QTLs (LOD > 3) were found across all chromosomes. Eight QTLs were detected under control, 9 for EPPN, 13 for EPPO conditions, 3 for C08, and 12 for CD08. In addition, 13 QTLs and 7 QTLs appeared for NS (probit) and TG (probit), respectively, after subtraction of control and EPPN treatment from EPPO, CD08, and C08. Most QTLs were found on chromosome $2 \mathrm{H}$ between 109 and $182 \mathrm{cM}$ and on chromosome $7 \mathrm{H}$ between 63 and $122 \mathrm{cM}$. QTLs with the highest LOD scores were found for control $\left(\mathrm{T} 50 ; R^{2}=42.6\right.$; $\left.\mathrm{LOD}=17.7\right)$ on $7 \mathrm{H}$, for EPPN (T50; $\left.R^{2}=55.0 ; \mathrm{LOD}=25.1\right)$ on $7 \mathrm{H}$, for EPPO treatment $\left(\mathrm{AUC} ; R^{2}=25.5\right.$; LOD $\left.=11.5\right)$ on $3 \mathrm{H}$, for C08 (\%TG; $\left.R^{2}=17.9 ; \mathrm{LOD}=7.3\right)$ on $2 \mathrm{H}$, and for $\mathrm{CD} 08\left(\% \mathrm{TG} ; R^{2}=42.9\right.$; $\mathrm{LOD}=16.3$ ) on $2 \mathrm{H}$.

Subtraction of control or EPPN treatment from EPPO, CD, and C08 is assumed to compensate for the effect of control 


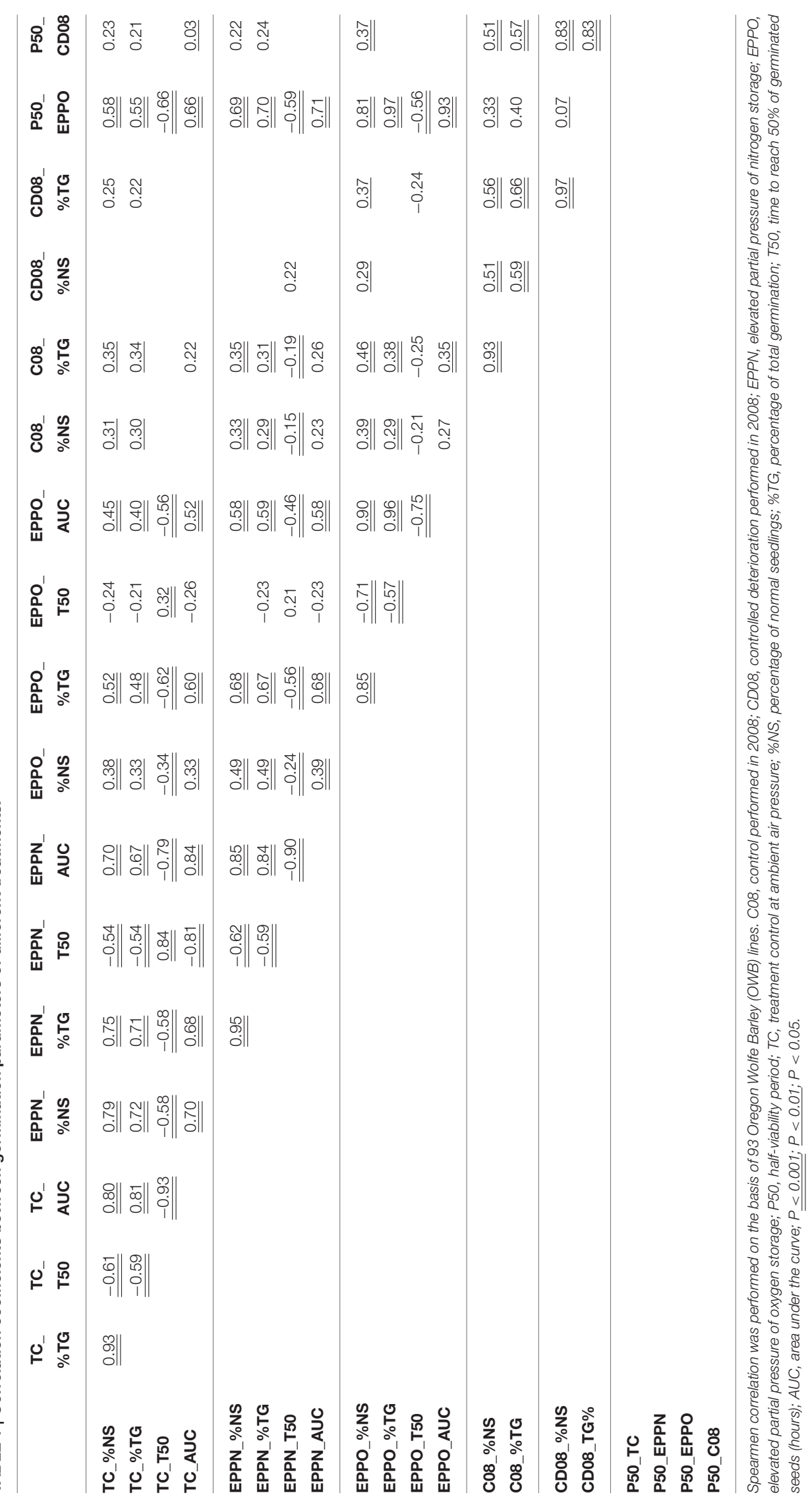




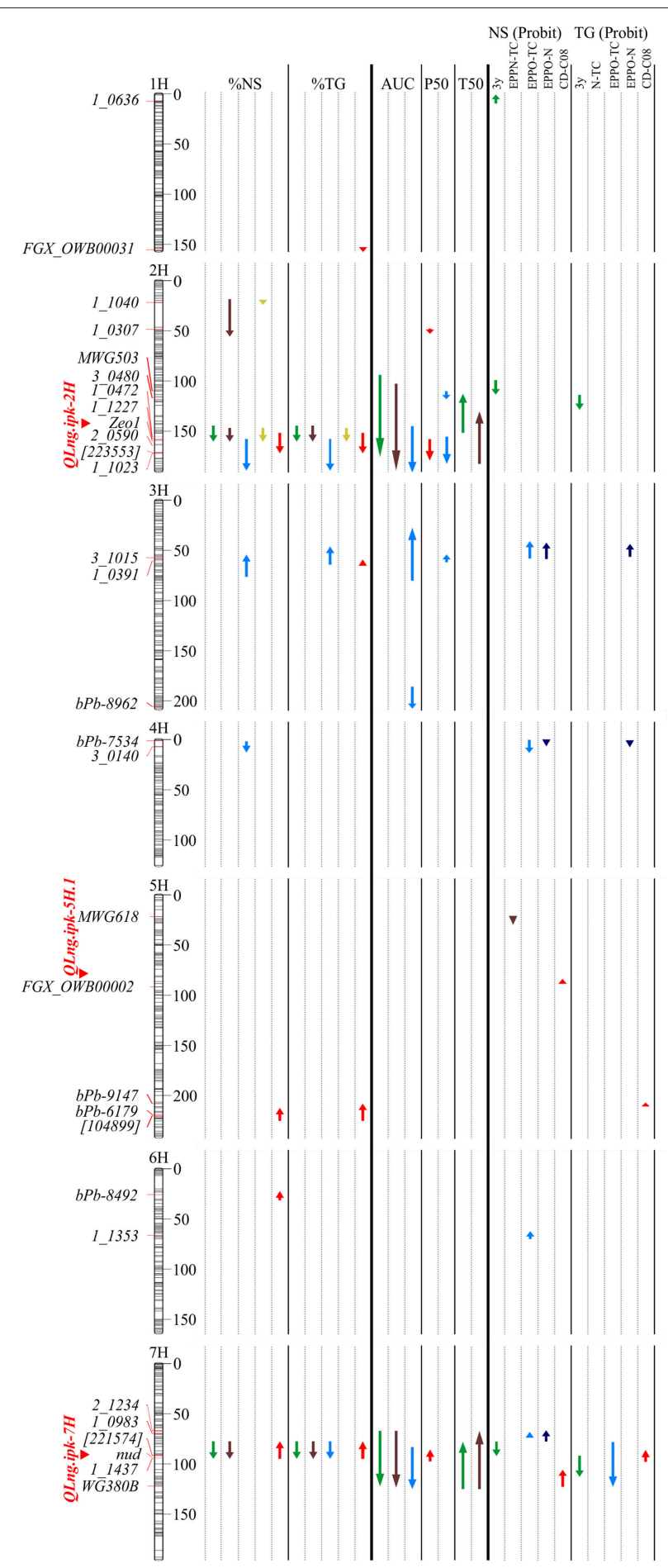

FIGURE 3 | Linkage map of Oregon Wolfe Barley (OWB) mapping population and quantitative trait locus (QTL) locations. QTLs detected under control conditions are marked in green (TC, treatment control, dark green; C08, control prepared in 2008, light green), those under nitrogen pressure (EPPN) in brown, under oxygen pressure (EPPO) in blue, and those after CD in 2008 red. Map distances are shown in cM. QTLs declared above a logarithm of odds (LOD) threshold of 3. Arrow lengths indicate QTL intervals having LOD > 3 and arrows up indicate that alleles came from the "OWB-D" parent and those down from the "OWB-R" parent. and high-pressure treatment and reveal treatment-specific loci. QTLs specific for EPPO were found on $3 \mathrm{H}, 4 \mathrm{H}$, and $6 \mathrm{H}$. On $5 \mathrm{H}$, two QTLs were found for $\mathrm{CD}(94.9$ and $207.7 \mathrm{cM})$ and one for EPPN $(21.4 \mathrm{cM})$ and, on $2 \mathrm{H}$, one QTL accounted for the differences after a 3-year storage period. Between 67 and $122 \mathrm{cM}$ on chromosome 7H, QTLs were detected under all treatments.

Interestingly, alleles with the greatest effect on most QTLs (39 out of 65) were contributed by the parent "OWB-R" showing the highest additive effects in CD08 and EPPO treatment with -2236.5 and -1290.1 for \%TG. The dominant parent "OWB-D" contributed the most alleles for QTLs on chromosome $3 \mathrm{H}$ after EPPO treatment and on $5 \mathrm{H}$ and $7 \mathrm{H}$ after $\mathrm{CD} 08$ treatment.

\section{DISCUSSION}

\section{High Nitrogen Pressure Induce White Seedling Appearance}

Anoxic conditions (produced by storage under 100\% nitrogen gas) are used in the food industry to preserve organic material from microbial growth (Van Campenhout et al., 2013). In the current experiment, EPPN was used as a second control to compensate for the potential effects of high pressure and pressure release. With respect to \%TG, AUC, and T50, high nitrogen pressure did not induce a significant effect compared to the control under ambient air pressure, but \%NS was significantly reduced, as a higher number of seedlings with white shoots and no roots were produced. A release of pressure from the tanks at the end of the storage period can create an increase in the volume of gases trapped in internal spaces, thereby causing physical disruption of tissues and producing seedlings with deformations. However, in our experiments with barley, the frequency of seedlings with deformations was not increased. A possible explanation for the white seedlings is nitrogen conversion to nitrogen oxides, which affect metabolism, oxidation state, or signaling (Bethke et al., 2007). This might also explain the observed dormancy alleviation for some lines, as indicated by an increase in the \%NS.

\section{Detrimental Effects of EPPO on Seed Germination}

Oxygen is essential for the survival of most living organisms under moist conditions, as it is required for aerobic respiration. With desiccation tolerant organisms such as seeds, respiration activity ceases under dry conditions and they can survive without oxygen. Molecular oxygen can have a detrimental effect through the formation of super oxide which reacts with almost all organic components and damages DNA, proteins and lipids, resulting in membrane leakage and cell death (Van Breusegem and Dat, 2006). The effect of high oxygen pressure was investigated by Caldwell (1964), who found that dry bean and pea seeds are able to tolerate high atmospheric oxygen pressure whereas imbibed beans and peas cannot. In our experiments, the dry barley seeds under investigation responded to high-pressure oxygen treatment with a considerable reduction in germination performance. After 9 weeks of dry storage under $\sim 18 \mathrm{MPa}$ oxygen, the \%NS and \% TG were reduced by 67 and $52 \%$ and 
TABLE 2 | Quantitative trait locus (QTL) analysis of the germination performance of the Oregon Wolfe Barley (OWB) mapping population after experimental aging methods.

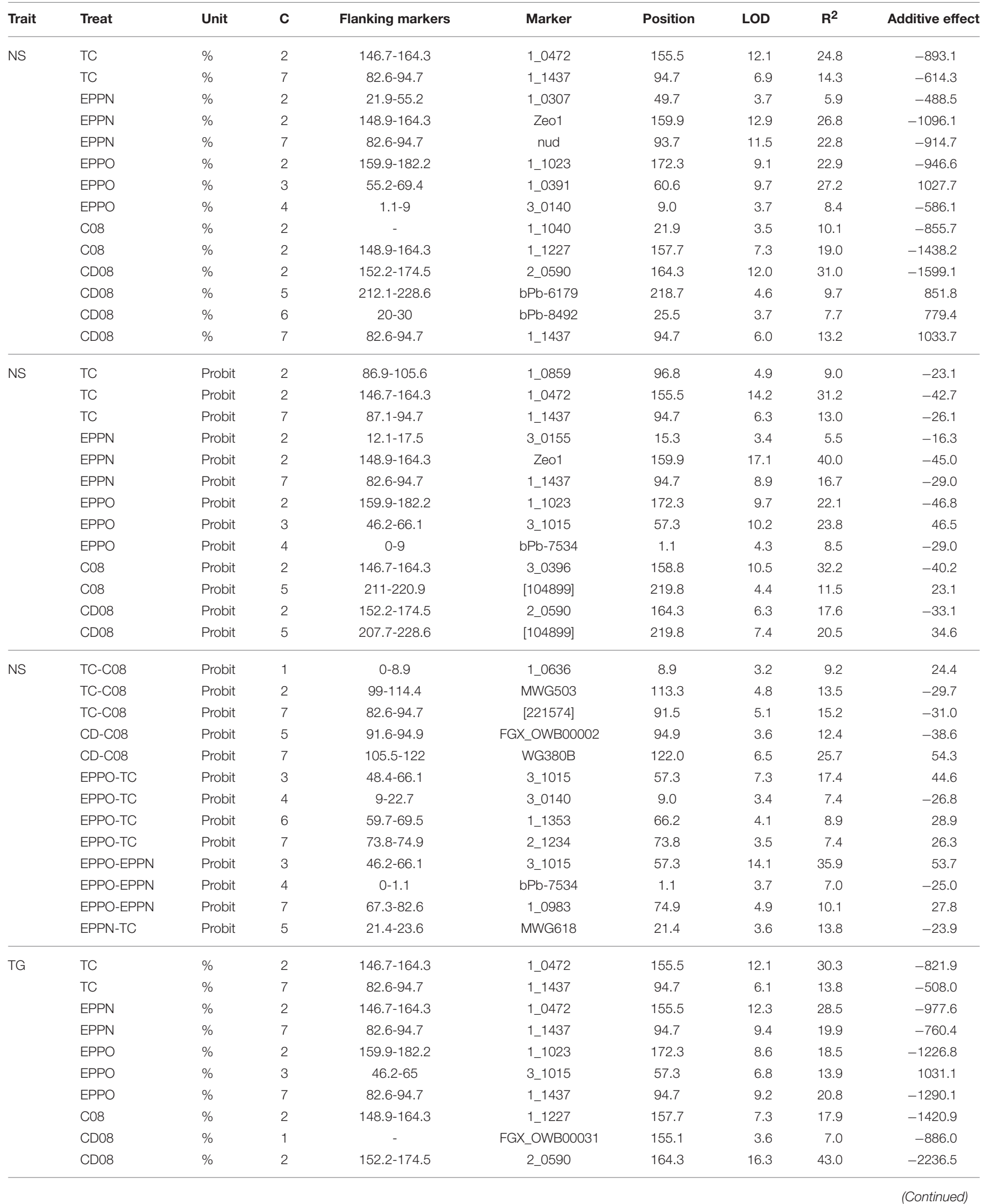


TABLE 2 | Continued

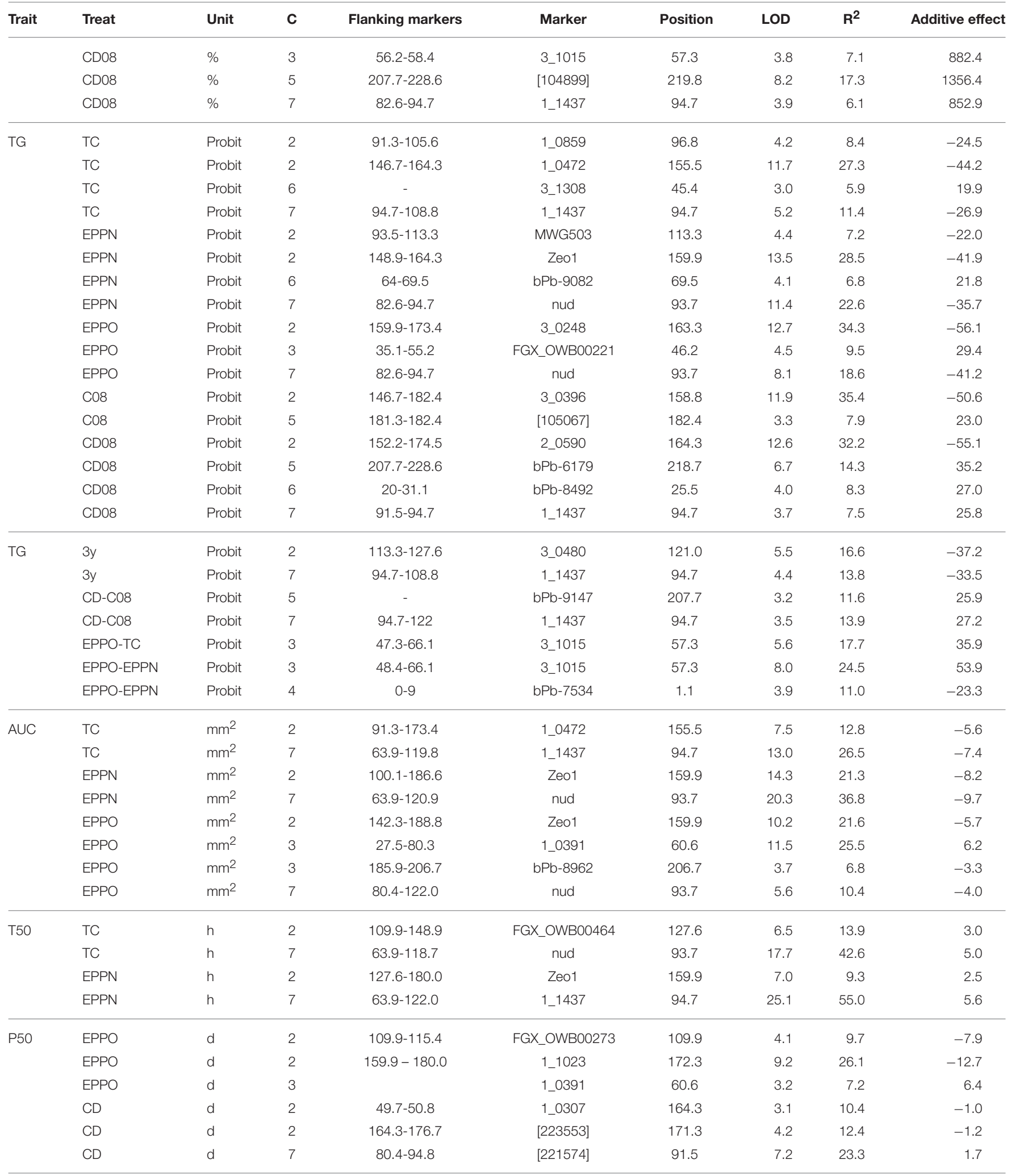

QTLs declared above a logarithm of odds (LOD) of 3. Positive additive effects indicate a contribution by "OWB-D." C08, control performed in 2008; CD08, controlled deterioration performed in 2008; EPPN, elevated partial pressure of nitrogen storage; EPPO, elevated partial pressure of oxygen storage; \%NS, percentage of normal seedlings; \%TG, percentage of total germination; TC, treatment control at ambient air pressure; T50, time to reach 50\% of germinated seeds (hours); AUC, area under the curve; P50, half-viability period. Chr, chromosome; $R^{2}$, proportion of explained phenotypic variance (\%); $C M$, centiMorgan. -, before the additive effect of the allele indicates that the larger value allele came from the "OWB-R"; +, before the additive effect of the allele indicates that the larger value allele came from the "OWB-D" parent. 
the time to $50 \%$ germination almost doubled. This increased deterioration confirms the deleterious effects of oxygen on seeds.

\section{Aging Treatment Affects Seed Deterioration}

The reduction in total number of germinating seeds indicates that barley seeds deteriorate more after 3 days of CD treatment than after 63 days of EPPO treatment. At the higher moisture content used in the $\mathrm{CD}$, the fluidity of intercellular glasses increases (Walters et al., 2010) and biochemical processes are accelerated (Lehner et al., 2008). The EPPO approach intends to use seed equilibrated to ambient moisture and temperature conditions (here $20^{\circ} \mathrm{C}$ and $40 \% \mathrm{RH}$ ) and are assumed, according to Walters et al. (2005a), in the glassy state. As the glass-phase transition temperature is assumed to be hardly affected by higher gas pressure (Groot et al., 2012), seeds might remain in the glassy, metabolically inactive (Fernández-Marín et al., 2013) state during EPPO storage and deterioration is caused by oxygen. Groot et al. (2012) demonstrated that the EPPO approach might simulate ambient storage better than $\mathrm{CD}$, as tocopherol content was linearly reduced with EPPO storage time, but not with CD, when seed quality declined.

\section{Linked Markers and Genes Indicate Relationship between Morphology, Stress Response Mechanism, and Seed Deterioration}

In general, seed deterioration is affected by many factors, which pop up during seed development, ripening, threshing, cleaning and storage. This activates a plethora of mechanisms on cellular level as exemplified by the functional annotation of genes (Supplementary Table 2). However, most QTLs were found with two previously defined areas termed QLng.ipk-2H and QLng.ipk$7 H$ (Nagel et al., 2009; Figure 3) and confirmed an association with the genetic panel of 175 diverse barley genotypes (Nagel et al., 2015). The QTL area on $2 \mathrm{H}$ estimated between 110 and 172 cM influences 21 traits, including plant height, spike length, grain number and grain yield. Thereby, the parent "OWB-D" provides dominant alleles to the progeny, is characterized by shorter plants (Chutimanitsakun et al., 2011) and is more sensitive to seed deterioration as "OWB-R". In agreement with this the detected Zeo1 gene determines plant height, spike compactness and kernel width (Franckowiak et al., 1997) caused by polymorphisms in the microRNA172 (miR172)-binding site of the barley ortholog of an APELATA2 transcription factor (HvAP2). It is indicated that changes result in altered timing of developmental events (Houston et al., 2013) which might affect seed quality and deterioration behavior. Further, in the centromeric region of $7 \mathrm{H}$ from 73 to $95 \mathrm{cM}, 18$ QTLs were identified. The nud gene, an ethylene response family transcription factor gene responsible for naked/covered caryopsis (Franckowiak and Konishi, 1997) is also in this area. It further controls the lipid biosynthesis pathway in the inner side of the hull, generating the adhesion between lemma and palea (Taketa et al., 2008) and $\beta$-glucan content (Capo-Chichi et al., 2012).

Further annotation of gene functions in the QTL areas revealed the appearance of harpin-induced protein 1 family
(Gechev et al., 2004) and glutaredoxins (Rouhier et al., 2008) which indicate a relationship to abiotic and especially oxidative stress response mechanism and programmed cell death. Similar, the regulation of plant defense response against pathogens are demonstrated by the protein cysteine proteinase RD21a (Shindo et al., 2012), proline extensin-like receptor kinase 1 (PERK1; Silva and Goring, 2002), the disease resistance protein NBSLRR (Belkhadir et al., 2004) and the MAP kinase substrate (MKS1; Andreasson et al., 2005). Due to metabolic inactivation during storage we speculate that proteins, kinases and factors are activated during seed development or germination.

\section{Specialized QTLs for Tolerance to the Two Aging Treatments}

Germination after aging/storage is influenced by the initial germination which seeds show after harvest (Roberts, 1973). This effect is due to differences in susceptibility to pathogens, attraction for insects, and abiotic stress tolerances during seed production. Here, the effect of initial germination was mathematically omitted by the subtraction of control or EPPN from EPPO. This mathematical procedure elucidated and/or confirmed QTLs for EPPO tolerance on $3 \mathrm{H}, 4 \mathrm{H}$, and $6 \mathrm{H}$, for $\mathrm{CD} 08$ on $5 \mathrm{H}$, and dormancy release on $2 \mathrm{H}$ calculated by differences between control in 2008 and the treatment control in 2011 (3 years). The QTL for CD08 shows colinearity (Stein et al., 2007) to strong and highly significant QTLs found for rice seed deterioration (Sasaki et al., 2005, 2015). This region contains a gene, annotated as encoding trehalose-6-phosphate phosphatase, which can be used to manipulate abiotic stress tolerance in rice (Sasaki et al., 2005).

Biochemical pathways and deterioration processes are influenced by water activity (seed moisture level), temperature and atmosphere. In agreement with this, we observed QTLs in response to both EPPO and CD storage, as well QTLs expressed as a results of genetic variation in longevity under either EPPO or $\mathrm{CD}$ storage conditions. This indicates the causes of deterioration under CD and storage under EPPO are partly similar and partly distinct controlled.

\section{AUTHOR CONTRIBUTIONS}

$\mathrm{MN}, \mathrm{AB}$, and SG conceived and designed research. MN, JK, SP, and SG conducted experiments. MN and SG analyzed data. MN and SG wrote the manuscript. All authors read and approved the manuscript.

\section{FUNDING}

Financial support was provided by the EU "Seventh Framework Programme" grant EcoSeed (No. 311840) and the Netherlands Ministry of Economic Affairs in the research project Improved seed storage, in the frame of the "Topconsortium Kennis en Innovatie Tuinbouw \& Uitgangsmaterialen program Meer met minder." The publication of this article was funded by the Open Access fund of the Leibniz Association. 


\section{ACKNOWLEDGMENTS}

We wish to acknowledge Annette Marlow, Stephanie Thumm and Peter Schreiber for experimental support and seed multiplication.

\section{REFERENCES}

Andreasson, E., Jenkins, T., Brodersen, P., Thorgrimsen, S., Petersen, N. H. T., $\mathrm{Zhu}$, S., et al. (2005). The MAP kinase substrate MKS1 is a regulator of plant defense responses. EMBO J. 24, 2579-2589. doi: 10.1038/sj.emboj.76 00737

Bailly, C. (2004). Active oxygen species and antioxidants in seed biology. Seed Sci. Res. 14, 93-107. doi: 10.1079/SSR2004159

Belkhadir, Y., Subramaniam, R., and Dangl, J. L. (2004). Plant disease resistance protein signaling: NBS-LRR proteins and their partners. Curr. Opin. Plant Biol. 7, 391-399. doi: 10.1016/j.pbi.2004.05.009

Bethke, P. C., Libourel, I. G. L., and Jones, R. L. (2007). "Nitric oxide in seed dormancy and germination," in Annual Plant Reviews: Vol. 27, Seed Development, Dormancy and Germination, eds K. J. Bradford and H. Nonogaki (Oxford: Blackwell Publishing Ltd), 153-175.

Buitink, J., and Leprince, O. (2004). Glass formation in plant anhydrobiotes: survival in the dry state. Cryobiology 48, 215-228. doi: 10.1016/j.cryobiol.2004.02.011

Buitink, J., and Leprince, O. (2008). Intracellular glasses and seed survival in the dry state. C. R. Biol. 331, 788-795. doi: 10.1016/j.crvi.2008.0 8.002

Caldwell, J. (1964). Effect of high pressures of pure oxygen on tissues. Nature 201, 514-515. doi: 10.1038/201514a0

Capo-Chichi, L., Kenward, K., Nyachiro, J., and Anyia, A. (2012). Nud locus and the effects on seedling vigour related traits for genetic improvement of hulless barley. J. Plant Sci. Mol. Breed. 1, 1-9. doi: 10.7243/2050-2389-1-2

Chutimanitsakun, Y., Nipper, R., Cuesta-Marcos, A., Cistué, L., Corey, A., Filichkina, T., et al. (2011). Construction and application for QTL analysis of a Restriction Site Associated DNA (RAD) linkage map in barley. BMC Genomics 12:4. doi: 10.1186/1471-2164-12-4

Costa, J. M., Corey, A., Hayes, P. M., Jobet, C., Kleinhofs, A., Kopisch-Obusch, A., et al. (2001). Molecular mapping of the Oregon Wolfe Barleys: a phenotypically polymorphic doubled-haploid population. Theoret. Appl. Genet. 103, 415-424. doi: $10.1007 /$ s001220100622

El-Maarouf-Bouteau, H., Mazuy, C., Corbineau, F., and Bailly, C. (2011). DNA alteration and programmed cell death during ageing of sunflower seed. J. Exp. Bot. 62, 5003-5011. doi: 10.1093/jxb/err198

Fernández-Marín, B., Kranner, I., Sebastián, M. S., Artetxe, U., Laza, J. M., Vilas, J. L., et al. (2013). Evidence for the absence of enzymatic reactions in the glassy state. A case study of xanthophyll cycle pigments in the desiccationtolerant moss Syntrichia ruralis. J. Exp. Bot. 64, 3033-3043. doi: 10.1093/jxb/e rt145

Franckowiak, D., and Konishi, T. (1997). Naked caryopsis. Barley Genet. Newsl. 26, 51-52.

Franckowiak, D., Lundqvist, U., and Konishi, T. (1997). New and revised names for barley genes. Barley Genet. Newsl. 26, 22-516.

Gechev, T. S., Gadjev, I. Z., and Hille, J. (2004). An extensive microarray analysis of AAL-toxin-induced cell death in Arabidopsis thaliana brings new insights into the complexity of programmed cell death in plants. Cell. Mol. Life Sci. 61, 1185-1197. doi: 10.1007/s00018-004-4067-2

Groot, S. P. C., De Groot, L., Kodde, J., and Van Treuren, R. (2014). Prolonging the longevity of ex situ conserved seeds by storage under anoxia. Plant Genet. Resour. 13, 18-26. doi: 10.1017/S14792621140 00586

Groot, S. P. C., Surki, A. A., De Vos, R. C. H., and Kodde, J. (2012). Seed storage at elevated partial pressure of oxygen, a fast method for analysing seed ageing under dry conditions. Ann. Bot. 110, 1149-1159. doi: 10.1093/aob/mcs198

\section{SUPPLEMENTARY MATERIAL}

The Supplementary Material for this article can be found online at: http://journal.frontiersin.org/article/10.3389/fpls.2016. 00388

Hampton, J. G., and Tekrony, D. M. (1995). Handbook of Vigour Test Methods. Zürich: International Seed Testing Association.

Hay, F. R., Adams, J., Manger, K., and Probert, R. (2008). The use of non-saturated lithium chloride solutions for experimental control of seed water content. Seed Sci. Technol. 36, 737-746. doi: 10.15258/sst.2008.36.3.23

Houston, K., Mckim, S. M., Comadran, J., Bonar, N., Druka, I., Uzrek, N., et al. (2013). Variation in the interaction between alleles of HvAPETALA2 and microRNA172 determines the density of grains on the barley inflorescence. Proc. Natl. Acad. Sci. U.S.A. 110, 16675-16680. doi: 10.1073/pnas.1311681110

ISTA (2008). International Rules for Seed Testing. Bassersdorf: International Seed Testing Association.

ISTA (2012). International Rules for Seed Testing. Bassersdorf: International Seed Testing Association.

Joosen, R. V. L., Kodde, J., Willems, L. A. J., Ligterink, W., Van Der Plas, L. H. W., and Hilhorst, H. W. M. (2010). GERMINATOR: a software package for highthroughput scoring and curve fitting of Arabidopsis seed germination. Plant J. 62, 148-159. doi: 10.1111/j.1365-313X.2009.04116.x

Kranner, I., Minibayeva, F. V., Beckett, R. P., and Seal, C. E. (2010). What is stress? Concepts, definitions and applications in seed science. New Phytol. 188, 655-673. doi: 10.1111/j.1469-8137.2010.03461.x

Lehner, A., Mamadou, N., Poels, P., Come, D., Bailly, C., and Corbineau, F. (2008). Changes in soluble carbohydrates, lipid peroxidation and antioxidant enzyme activities in the embryo during ageing in wheat grains. J. Cereal Sci. 47, 555-565. doi: $10.1016 /$ j.jcs.2007.06.017

Li, H. (2013). Aligning sequence reads, clone sequences and assembly contigs with BWA-MEM. arXiv:1303.3997 [q-bio.GN] [Online].

Li, H., Handsaker, B., Wysoker, A., Fennell, T., Ruan, J., Homer, N., et al. (2009). The Sequence Alignment/Map format and SAMtools. Bioinformatics 25, 2078-2079. doi: 10.1093/bioinformatics/btp352

Mascher, M., Muehlbauer, G. J., Rokhsar, D. S., Chapman, J., Schmutz, J., Barry, K., et al. (2013). Anchoring and ordering NGS contig assemblies by population sequencing (POPSEQ). Plant J. 76, 718-727. doi: 10.1111/tpj.12319

Nagel, M., and Börner, A. (2010). The longevity of crop seeds stored under ambient conditions. Seed Sci. Res. 20, 1-12. doi: 10.1017/S09602585099 90213

Nagel, M., Kranner, I., Neumann, K., Rolletschek, H., Seal, C. E., Colville, L., et al. (2015). Genome-wide association mapping and biochemical markers reveal that seed ageing and longevity are intricately affected by genetic background and developmental and environmental conditions in barley. Plant Cell Environ. 38, 1011-1022. doi: 10.1111/pce. 12474

Nagel, M., Vogel, H., Landjeva, S., Buck-Sorlin, G., Lohwasser, U., Scholz, U., et al. (2009). Seed conservation in ex-situ genebanks - genetic studies on longevity in barley. Euphytica 170, 1-10. doi: 10.1007/s10681-009-9975-7

Probert, R. J., Daws, M. I., and Hay, F. R. (2009). Ecological correlates of ex situ seed longevity: a comparative study on 195 species. Ann. Bot. 104, 57-69. doi: 10.1093/aob/mcp082

Quinlan, A. R., and Hall, I. M. (2010). BEDTools: a flexible suite of utilities for comparing genomic features. Bioinformatics 26, 841-842. doi: 10.1093/bioinformatics/btq.033

R Core Team (2015). R Foundation for Statistical Computing. Göttingen: R Core Team.

Rehman Arif, M. A., Nagel, M., Neumann, K., Kobiljski, B., Lohwasser, U., and Börner, A. (2012). Genetic studies of seed longevity in hexaploid wheat using segregation and association mapping approaches. Euphytica 186, 1-13. doi: 10.1007/s10681-011-0471-5

Roberts, E. H. (1960). The viability of cereal seed in relation to temperature and moisture: with eight figures in the text. Ann. Bot. 24, 12-31. 
Roberts, E. H. (1973). Predicting the storage life of seeds. Seed Sci. Technol. 1, 499-514.

Rouhier, N., Lemaire, S. D., and Jacquot, J.-P. (2008). The role of glutathione in photosynthetic organisms: emerging functions for glutaredoxins and glutathionylation. Annu. Rev. Plant Biol. 59, 143-166. doi: 10.1146/annurev.arplant.59.032607.092811

Sasaki, K., Fukuta, Y., and Sato, T. (2005). Mapping of quantitative trait loci controlling seed longevity of rice (Oryza sativa L.) after various periods of seed storage. Plant Breed. 124, 361-366. doi: 10.1111/j.1439-0523.2005.01109.x

Sasaki, K., Takeuchi, Y., Miura, K., Yamaguchi, T., Ando, T., Ebitani, T., et al. (2015). Fine mapping of a major quantitative trait locus, qLG-9, that controls seed longevity in rice (Oryza sativa L.). Theoret. Appl. Genet. 128, 769-778. doi: 10.1007/s00122-015-2471-7

The International Barley Genome Sequencing Consortium. (2012). A physical, genetic and functional sequence assembly of the barley genome. Nature 491, 711-716. doi: 10.1038/nature11543

Shindo, T., Misas-Villamil, J. C., Horger, A. C., Song, J., and Van Der Hoorn, R. A. L. (2012). A role in immunity for Arabidopsis cysteine protease RD21, the ortholog of the tomato immune protease C14. PLoS ONE 7:e29317. doi: 10.1371/journal.pone.0029317

Silva, N. F., and Goring, D. R. (2002). The proline-rich, extensin-like receptor kinase-1 (PERK1) gene is rapidly induced by wounding. Plant Mol. Biol. 50, 667-685. doi: 10.1023/A:1019951120788

Stein, N., Prasad, M., Scholz, U., Thiel, T., Zhang, H. N., Wolf, M., et al. (2007). A 1,000-loci transcript map of the barley genome: new anchoring points for integrative grass genomics. Theoret. Appl. Genet. 114, 823-839. doi: 10.1007/s00122-006-0480-2

Sun, W. Q., and Leopold, A. C. (1995). The Maillard reaction and oxidative stress during aging of soybean seeds. Physiol. Plant. 94, 94-104. doi: 10.1111/j.13993054.1995.tb00789.x

Taketa, S., Amano, S., Tsujino, Y., Sato, T., Saisho, D., Kakeda, K., et al. (2008). Barley grain with adhering hulls is controlled by an ERF family transcription factor gene regulating a lipid biosynthesis pathway. Proc. Natl. Acad. Sci. U.S.A. 105, 4062-4067. doi: 10.1073/pnas.0711034105

Van Breusegem, F., and Dat, J. F. (2006). Reactive oxygen species in plant cell death. Plant Physiol. 141, 384-390. doi: 10.1104/pp.106.078295

Van Campenhout, L., Maes, P., and Claes, J. (2013). Modified atmosphere packaging of Tofu: headspace gas profiles and microflora during storage. J. Food Process. Preserv. 37, 46-56. doi: 10.1111/j.1745-4549.2011.00612.x
Walters, C. (1998). Understanding the mechanisms and kinetics of seed aging. Seed Sci. Res. 8, 223-244. doi: 10.1017/S09602585000 0413X

Walters, C., Ballesteros, D., and Vertucci, V. A. (2010). Structural mechanics of seed deterioration: standing the test of time. Plant Sci. 179, 565-573. doi: 10.1016/j.plantsci.2010.06.016

Walters, C., Hill, L. M., and Wheeler, L. J. (2005a). Dying while dry: kinetics and mechanisms of deterioration in desiccated organisms. Integr. Comp. Biol. 45, 751-758. doi: 10.1093/icb/45.5.751

Walters, C., Wheeler, L. M., and Grotenhuis, J. M. (2005b). Longevity of seeds stored in a genebank: species characteristics. Seed Sci. Res. 15, 1-20. doi: 10.1079/SSR2004195

Wang, S., Basten, C. J., and Zeng, Z.-B. (2011). Windows QTL Cartographer 2.5. Department of Statistics, North Carolina State University, Raleigh, NC. Available online at: http://statgen.ncsu.edu/qtlcart/WQTLCart.htm.

Waterworth, W. M., Masnavi, G., Bhardwaj, R. M., Jiang, Q., Bray, C. M., and West, C. E. (2010). A plant DNA ligase is an important determinant of seed longevity. Plant J. 63, 848-860. doi: 10.1111/j.1365-313X.2010.04 285.x

Wolfe, R. I., and Franckowiak, J. D. (1991). Multiple dominant and recessive genetic marker stocks in spring barley. Barley Genet. Newsl. 20, 117-121.

Zeng, Z. B. (1994). Precision mapping of quantitative trait loci. Genetics 136, $1457-1468$.

Conflict of Interest Statement: The authors declare that the research was conducted in the absence of any commercial or financial relationships that could be construed as a potential conflict of interest.

The reviewer PK and handling Editor declared their shared affiliation, and the handling Editor states that the process nevertheless met the standards of a fair and objective review.

Copyright (c) 2016 Nagel, Kodde, Pistrick, Mascher, Börner and Groot. This is an open-access article distributed under the terms of the Creative Commons Attribution License (CC BY). The use, distribution or reproduction in other forums is permitted, provided the original author(s) or licensor are credited and that the original publication in this journal is cited, in accordance with accepted academic practice. No use, distribution or reproduction is permitted which does not comply with these terms. 